\title{
Isolated subdural hematomas in mild traumatic brain injury. Part 1: the association between radiographic characteristics and neurosurgical intervention
}

\author{
Alessandro Orlando, MPH, ${ }^{1,4-6}$ A. Stewart Levy, MD, ${ }^{3}$ Benjamin A. Rubin, MD, ${ }^{2}$ Allen Tanner, MD, ${ }^{6}$ \\ Matthew M. Carrick, MD, ${ }^{5}$ Mark Lieser, MD, ${ }^{7}$ David Hamilton, MD, ${ }^{6}$ Charles W. Mains, MD, ${ }^{4}$ and \\ David Bar-Or, MD ${ }^{1,4-6,8}$
}

${ }^{1}$ Trauma Research Department and 2Department of Neurosurgery, Swedish Medical Center, Englewood, Colorado; ${ }^{3}$ Department of Neurosurgery and ${ }^{4}$ Trauma Research Department, St. Anthony Hospital, Lakewood, Colorado; ${ }^{5}$ Trauma Research Department, Medical City Plano, Plano, Texas; ${ }^{6}$ Trauma Research Department, Penrose Hospital, Colorado Springs, Colorado; ${ }^{7}$ Trauma Services Department, Research Medical Center, Kansas City, Missouri; and ${ }^{8}$ Rocky Vista University College of Osteopathic Medicine, Parker, Colorado

OBJECTIVE Isolated subdural hematomas (iSDHs) are one of the most common intracranial hemorrhage (ICH) types in the population with mild traumatic brain injury (mTBl; Glasgow Coma Scale score 13-15), account for $66 \%-75 \%$ of all neurosurgical procedures, and have one of the highest neurosurgical intervention rates. The objective of this study was to examine how quantitative hemorrhage characteristics of iSDHs in patients with $\mathrm{mTBI}$ at admission are associated with subsequent neurosurgical intervention.

METHODS This was a 3.5-year, retrospective observational cohort study at a Level I trauma center. All adult trauma patients with $\mathrm{mTBI}$ and iSDHs were included in the study. Maximum length and thickness (in $\mathrm{mm}$ ) of acute SDHs, the presence of acute-on-chronic SDH, mass effect, and other hemorrhage-related variables were double-data entered; discrepant results were adjudicated after a maximum of 4 reviews. Patients with coagulopathy, skull fractures, no acute hemorrhage, a non-SDH ICH, or who did not undergo imaging on admission were excluded. The primary outcome was neurosurgical intervention (craniotomy, burr hole, catheter drainage of $\mathrm{SDH}$, placement of intracranial pressure monitor, shunt, or ventriculostomy). Multivariate stepwise logistic regression was used to identify significant covariates and to assess interactions.

RESULTS A total of 176 patients were included in our study: 28 patients did and 148 patients did not receive a neurosurgical intervention. Increasing head Abbreviated Injury Scale score was significantly associated with neurosurgical interventions. There was a strong correlation between the first 3 reviews on maximum hemorrhage length $\left(R^{2}=0.82\right)$ and maximum hemorrhage thickness $\left(R^{2}=0.80\right)$. The neurosurgical intervention group had a mean maximum SDH length and thickness that were $61 \mathrm{~mm}$ longer and $13 \mathrm{~mm}$ thicker than those of the nonneurosurgical intervention group $(p<0.001$ for both). After adjusting for the presence of an acute-on-chronic hemorrhage, for every 1-mm increase in the thickness of an iSDH, the odds of a neurosurgical intervention increase by $32 \%(95 \% \mathrm{Cl} 1.16-1.50)$. There were no interventions for any SDH with a maximum thickness $\leq 5 \mathrm{~mm}$ on initial presenting scan.

CONCLUSIONS This is the first study to quantify the odds of a neurosurgical intervention based on hemorrhage characteristics in patients with an iSDH and mTBI. Once validated in a second population, these data can be used to better inform patients and families of the risk of future neurosurgical intervention, and to evaluate the necessity of interhospital transfers.

https://thejns.org/doi/abs/10.3171/2018.1.JNS171884

KEYWORDS neurosurgical intervention; neurosurgery; mild; traumatic brain injury; subdural hematoma; epidemiology

ABBREVIATIONS AIS = Abbreviated Injury Scale; $A O C=$ acute-on-chronic; $A U R O C=$ area under the receiver operating characteristic; ED = emergency department; EDH = epidural hematoma; GCS = Glasgow Coma Scale; ICH = intracranial hemorrhage; iSDH = isolated subdural hematoma; ISS = Injury Severity Score; IVH = intraventricular hemorrhage; $L O C=$ loss of consciousness; LOS = length of stay; mTBI = mild traumatic brain injury; $S A H$ = subarachnoid hemorrhage.

SUBMITTED August 3, 2017. ACCEPTED January 4, 2018.

INCLUDE WHEN CITING Published online June 15, 2018; DOI: 10.3171/2018.1.JNS171884. 
$\mathrm{T}$ Raumatic brain injuries (TBIs) vary in severity, with each severity category carrying a different risk for neurosurgical intervention. Despite this understanding, most patients suffering a TBI with intracranial hemorrhage (ICH) identified on CT scans of the head are treated equally in terms of requisite neurosurgical coverage, regardless of the severity of injury, leading to inefficiencies in hospital resource utilization and potentially unnecessary interhospital transfers. Of the 1.7 million annual TBIs in the US, ${ }^{5}$ approximately 127,500 are mild (mTBI; Glasgow Coma Scale [GCS] score of 13-15) and are accompanied by an $\mathrm{ICH}^{18,28}$ Due to the potential for neurological decline and subsequent need for neurosurgical intervention, these patients are most often evaluated by a neurosurgeon, or transferred to a hospital with neurosurgical coverage; for some patients, this results in a lengthy and costly interhospital transfer.

The overall neurosurgical intervention rate for patients with an mTBI and ICH ranges from $0 \%$ to $9 \% ; 1,2,4,10,13,29$, 30,32-34 however, there is also considerable variation in the intervention rate according to the specific ICH type. In a 2015 study, Sweeney and colleagues examined patients with isolated mTBI, excluding those with skull fractures and penetrating trauma. ${ }^{32}$ Across 6 years of data from the National Trauma Data Bank, they observed an overall neurosurgical intervention rate of $8.8 \%$, with various ICH types having neurosurgical intervention rates ranging from $1.5 \%$ for subarachnoid hemorrhages (SAHs) to $17.6 \%$ for epidural hematomas (EDHs). In a similar multicenter study examining patients with mTBI and ICHexcluding patients with coagulopathies-Orlando et al. observed an overall neurosurgical intervention rate of $6.7 \%$, with rates ranging from $0.95 \%$ for isolated SAHs to $15.5 \%$ for subdural hematomas (SDHs). ${ }^{20}$ There are differences in the risk for neurosurgical intervention based on the specific type of ICH in the mTBI population, yet management guidelines treat all ICHs equally, requiring neurosurgical consultations, often necessitating transfer to a facility with neurosurgical coverage, for all mTBIs with an ICH.

A question repeatedly posed in the TBI literature is whether a neurosurgical consultation, or a requirement for neurosurgical coverage, is necessary for all patients with an mTBI and ICH. ${ }^{2,3,8,11,14,17,36}$ Unfortunately, we do not have enough of an understanding of the risk for neurosurgical intervention to create management guidelines addressing particular ICH types in patients with mTBI. ${ }^{9}$ In order to better understand the risk, we must collect detailed information about each injury and assess the influence of each variable on the risk of neurosurgical intervention through predictive modeling. Because all ICH types in the mTBI population carry different physiological mechanisms and neurosurgical intervention rates, it is necessary to create predictive models for each ICH type.

Isolated SDHs are a prime target for the creation of predictive models for neurosurgical intervention, because they are one of the most common ICH types in the mTBI population, account for $66 \%-75 \%$ of all neurosurgical procedures, and have one of the highest neurosurgical intervention rates. ${ }^{19,20,32}$ The objective of Part 1 of this study was to examine how quantitative hemorrhage character- istics of isolated subdural hematomas (iSDHs) in patients with mTBI at admission are associated with subsequent neurosurgical intervention.

\section{Methods \\ Study Site and Patient Population}

This was a single-center retrospective observational cohort study of adult trauma patients consecutively admitted over 3.5 years at a Level I trauma center. Data were collected through a combination of registry data and chart abstraction. Patients were included if they presented with an mTBI (emergency department [ED] GCS score 13-15) and had a head CT positive for SDH (ICD-9 852.2-852.39). Patients were excluded from the study if they presented with 1) skull fractures, because the ICH type cannot be determined from skull fracture-related ICD-9 codes; 2) coagulopathy on admission; 3) no acute hemorrhage; 4) a hemorrhage other than an SDH, not including intraventricular hemorrhages (IVHs); 5) no radiological imaging of hemorrhage on admission; or 6) only radiological imaging that was obtained after a neurosurgical procedure. This study was reviewed and approved by the institutional review board and was granted a waiver of consent.

\section{Outcomes and Covariates}

The primary outcome of this study was execution of a neurosurgical procedure (craniotomy/craniectomy/craniostomy, burr hole, catheter drainage of SDH, placement of intracranial pressure monitor, shunt, or ventriculostomy) or a neurosurgeon-recommended neurosurgical procedure. If a neurosurgeon recommended an operation but did not proceed because the patient or family refused, the patient was placed on comfort care, or there were other circumstances preventing a neurosurgical operation, the patient was still considered to have received neurosurgical intervention for this study. The objective of this study was to characterize how iSDHs are associated with the recommendation or necessity of a neurosurgeon to operate; we did not wish to introduce patient or family bias.

The secondary outcome was in-hospital mortality. Covariates were as follows: age, sex, interhospital transfer status, mechanism of injury (fall, motor vehicle accident, and other), ED GCS score, severe head injury (maximum head Abbreviated Injury Scale [AIS] score $\geq 4$ ), Injury Severity Score (ISS $0-15, \geq 16$ ), normal ED blood pressure (systolic $<120 \mathrm{~mm} \mathrm{Hg}$ and diastolic $<80 \mathrm{~mm} \mathrm{Hg}$ ), normal ED respiratory rate (12-16 breaths/min), normal ED pulse (60-100 beats/min), normal ED body temperature $\left(36.5^{\circ} \mathrm{C}-37.2^{\circ} \mathrm{C}\right)$, normal ED blood oxygen levels $(95 \%-$ $100 \%$ saturation), ED disposition, comorbid conditions (obesity, alcoholism, diabetes mellitus, and hypertension), hospital length of stay (LOS, days), and hospital disposition.

\section{Radiological Data Abstraction}

The following were double-data entered from the electronic medical record for each patient encounter: clinical presentation signs and symptoms (concomitant concussion, nausea, vomiting, headache, dizziness, poor concentration, fatigue, seizures, irritability, rhinorrhea, otorrhea, 
hemotympanum, raccoon eyes, no pupil response, hypothermia, hypoxia, and postinjury loss of consciousness [LOC]); hemorrhage lobe involvement (falx and tentorial hemorrhages were not assigned a lobe of involvement); and hemorrhage status on follow-up CT (obtained from CT reports-completely resolved, resolving, stable, increased, and no follow-up CT). The presence of acute-on-chronic (AOC) hemorrhage, IVH, mass effect, and midline shift was obtained from CT reports.

Hemorrhage measurements were also double-data entered using admission CT or MRI scans. If initial imaging was obtained at an outlying facility, measurements were taken from that facility's images. Maximum hemorrhage length was measured from the most anterior to the most posterior location, using transverse/axial CT or MR images, with at least 4 measurements used to obtain the length. Maximum hemorrhage thickness was measured perpendicularly from the cortex to the skull, also using transverse/axial CT or MR images. Measurements of maximum thickness and maximum length were allowed to have been obtained from different slices of the head CT or MR image. Measurements were taken using the ruler tool in the picture archiving and communication system (PACS) and rounded to the nearest tenths' place. Special attention was paid to not obtain thickness measurements too high on the skull, due to artificially larger thickness measurements secondary to the increasingly tangential nature of axial slices at higher levels. Additionally, tentorial SDHs were not measured, because coronal imaging was not available for all patients. If a lesion presented as an AOC hemorrhage, both the acute and chronic portions of the hemorrhage were measured as one.

If maximum hemorrhage length was discrepant by $>15$ $\mathrm{mm}$ between reviewers, or if maximum hemorrhage thickness was discrepant by $>5 \mathrm{~mm}$, a third reviewer (A.O.) measured and replaced the most discrepant measurement, and the two measurements were averaged. If there continued to be a discrepancy after the third measurement, a final measurement was taken by a fourth reviewer (A.S.L.) and that was used as the final value. The final maximum hemorrhage measurement was the average of two measurements, unless a fourth review was required.

\section{Statistical Analysis}

Univariate analyses performed using chi-square tests or Fisher's exact tests, and 2-sample t-tests and Wilcoxon rank-sum tests examined the association between all covariates and outcome measures. Pearson correlation coefficients were used to assess interrater agreement on the measurement of the following two continuous variables: maximum hemorrhage thickness and maximum hemorrhage length. Depending on the equality of variances, the pooled or Satterthwaite $\mathrm{p}$ values are reported for the 2-sample t-tests. Cochran-Armitage trend tests examined the proportion of patients with neurosurgical intervention and the number of lobes involved in hemorrhage (p-trend). Stepwise multivariable logistic regression models were built to analyze the independent association covariates and outcome measures. Entry and exit criteria for these models were 0.20 and 0.15 , respectively. The presence of effect modification was examined for all variables includ- ed in the final models. Area under the receiver operating characteristic (AUROC) curves and Hosmer-Lemeshow goodness-of-fit statistics are reported for all adjusted logistic regression models; 95\% confidence intervals for AUROC curves were calculated using the binomial exact method. All statistical analyses were 2-tailed and had an alpha value of 0.05. SAS software, version 9.3 (SAS Institute), was used for all analyses.

\section{Results}

There were 176 patients included in this study. Overall, the majority of patients were 65 years or older, and male (Table 1). Sixty percent of patients were transferred from an outlying hospital. According to the maximum head AIS score, $39 \%$ suffered a serious head injury (ED AIS score $\geq 4$ ); however, $95 \%$ had a GCS score of 14 or 15 . Ninetyeight percent of non-GCS-15 scores were attributable to decreased verbal functioning. Furthermore, almost half of the patients had polytrauma, reflected by an ISS $>15$. Hypertension was the most prevalent comorbid condition $(52 \%),>60 \%$ of patients reported headache in the ED, and $39 \%$ reported an LOC after injury. The top three lobes involved were the frontal, temporal, and parietal lobes (Table 2). Most patients presented with acute hemorrhages and showed no signs of midline shift, and only 2 patients presented with IVH.

Of the 176 patients included in the study, 167 had measured hemorrhages; 9 patients only had tentorial SDHs and were not measured. There was a strong correlation between the first two reviewers on maximum hemorrhage length $\left(\mathrm{R}^{2}=0.82\right)$ and maximum hemorrhage thickness $\left(\mathrm{R}^{2}=0.80\right)$. After a third review, 49 length measurements and 12 thickness measurements continued to be discrepant, requiring a fourth and final review. The average measurement difference between the final review and the third review's average was $9.5 \mathrm{~mm}$ for length and $4.5 \mathrm{~mm}$ for thickness. The median maximum length for iSDHs was $94.5 \mathrm{~mm}$, and the median maximum thickness was 7.0 $\mathrm{mm}$. The largest maximum length and thickness were $221.0 \mathrm{~mm}$ and $35.3 \mathrm{~mm}$, respectively (Table 3 ).

The neurosurgical intervention rate in our iSDH population with mTBI was $15.9 \%$. When comparing demographic and clinical presentation characteristics between neurosurgical intervention groups, there were only two significant differences: the nonneurosurgical intervention group had a significantly larger proportion of patients with a postinjury LOC, and the neurosurgical intervention group had a significantly larger proportion of patients with higher head AIS scores (Table 1).

There were several differences between neurosurgical intervention groups when comparing SDH radiological characteristics (Table 2). The neurosurgical intervention group had a significantly larger proportion of patients with hemorrhages involving the frontal, temporal, parietal, and occipital lobes-essentially holohemispheric SDHs, whereas the nonneurosurgical intervention group had a significantly larger proportion of patients with SDH occurring along the falx, and showed a trend toward having a significantly larger proportion of patients with tentorial SDHs compared to the neurosurgical intervention group. 
TABLE 1. Demographic differences between outcome groups in patients with $\mathrm{mTBI}$ and iSDH

\begin{tabular}{|c|c|c|c|c|}
\hline Patient Characteristics & $\begin{array}{l}\text { Overall, } \\
\mathrm{N}=176\end{array}$ & $\begin{array}{l}\text { No Neurosurgical Intervention, } \\
\qquad n=148\end{array}$ & $\begin{array}{l}\text { Neurosurgical Intervention, } \\
n=28\end{array}$ & $\begin{array}{c}p \\
\text { Value }\end{array}$ \\
\hline Male & $93(53 \%)$ & $78(53 \%)$ & $15(54 \%)$ & 0.93 \\
\hline Age, yrs & & & & 0.61 \\
\hline $18-64$ & $64(36 \%)$ & $55(37 \%)$ & $9(32 \%)$ & \\
\hline$\geq 65$ & $112(64 \%)$ & $93(63 \%)$ & $19(68 \%)$ & \\
\hline Interhospital transfer & $106(60 \%)$ & $90(61 \%)$ & $16(57 \%)$ & 0.72 \\
\hline Head AIS score & & & & 0.02 \\
\hline 2 & $5(3 \%)$ & $5(3 \%)$ & 0 & \\
\hline 3 & $103(59 \%)$ & $84(57 \%)$ & $19(68 \%)$ & \\
\hline 4 & $56(32 \%)$ & $52(35 \%)$ & $4(14 \%)$ & \\
\hline 5 & $12(7 \%)$ & $7(5 \%)$ & $5(18 \%)$ & \\
\hline ED GCS score & & & & 0.22 \\
\hline 13 & $9(5 \%)$ & $7(5 \%)$ & $2(7 \%)$ & \\
\hline 14 & $37(21 \%)$ & $28(19 \%)$ & $9(32 \%)$ & \\
\hline 15 & $130(74 \%)$ & $113(76 \%)$ & $17(61 \%)$ & \\
\hline ISS & & & & 0.12 \\
\hline $1-15$ & $96(55 \%)$ & $77(52 \%)$ & $19(68 \%)$ & \\
\hline$\geq 16$ & $80(45 \%)$ & $71(48 \%)$ & $9(32 \%)$ & \\
\hline Normal ED pulse: $60-100$ beats/min* & $142(81 \%)$ & $119(80 \%)$ & $23(85 \%)$ & 0.56 \\
\hline Normal ED blood pressure: SBP $<120$ \& DBP $<80$ mm Hg $\dagger$ & $25(14 \%)$ & $21(14 \%)$ & $4(15 \%)$ & $>0.99$ \\
\hline Normal ED respiratory rate: $12-16$ breaths/min $\ddagger$ & $74(43 \%)$ & $66(45 \%)$ & $8(31 \%)$ & 0.18 \\
\hline Normal ED temp: $36.5^{\circ} \mathrm{C}-37.2^{\circ} \mathrm{C} \S$ & $116(72 \%)$ & $103(74 \%)$ & $13(62 \%)$ & 0.27 \\
\hline \multicolumn{5}{|l|}{ Comorbid conditions } \\
\hline Hypertension & $91(52 \%)$ & $75(51 \%)$ & $16(57 \%)$ & 0.53 \\
\hline Obesity & $13(7 \%)$ & $12(8 \%)$ & $1(4 \%)$ & 0.70 \\
\hline Alcoholism & $25(14 \%)$ & $22(15 \%)$ & $3(11 \%)$ & 0.77 \\
\hline Diabetes mellitus & $29(16 \%)$ & $27(18 \%)$ & $2(7 \%)$ & 0.18 \\
\hline \multicolumn{5}{|l|}{ Clinical presentation } \\
\hline Nausea & $41(23 \%)$ & $34(23 \%)$ & $7(25 \%)$ & 0.82 \\
\hline Vomiting & $22(13 \%)$ & $18(12 \%)$ & $4(14 \%)$ & 0.76 \\
\hline Headache & $108(61 \%)$ & $93(63 \%)$ & $15(54 \%)$ & 0.36 \\
\hline Dizziness & $30(17 \%)$ & $24(16 \%)$ & $6(21 \%)$ & 0.58 \\
\hline Poor concentration & $36(20 \%)$ & $30(20 \%)$ & $6(21 \%)$ & 0.89 \\
\hline Fatigue & $9(5 \%)$ & $6(4 \%)$ & $3(11 \%)$ & 0.16 \\
\hline Seizures & $9(5 \%)$ & $8(5 \%)$ & $1(4 \%)$ & $>0.99$ \\
\hline Irritability & $10(6 \%)$ & $10(7 \%)$ & 0 & 0.37 \\
\hline Rhinorrhea & $1(1 \%)$ & $1(1 \%)$ & 0 & $>0.99$ \\
\hline Otorrhea & 0 & 0 & 0 & NA \\
\hline Hemotympanum & 0 & 0 & 0 & NA \\
\hline Raccoon eyes & $5(3 \%)$ & $5(3 \%)$ & 0 & $>0.99$ \\
\hline No pupil response & 0 & 0 & 0 & NA \\
\hline Hypothermia & 0 & 0 & 0 & NA \\
\hline Hypoxia & 0 & 0 & 0 & NA \\
\hline Postinjury LOC & $68(39 \%)$ & $65(44 \%)$ & $3(11 \%)$ & $<0.001$ \\
\hline
\end{tabular}

AIS = Abbreviated Injury Scale; DBP = diastolic blood pressure; NA = not applicable; SBP = systolic blood pressure.

Boldface type indicates statistical significance. Values were missing for some of the ED data; therefore, those denominators are different from the group and overall totals, as listed below.

* One patient in the neurosurgical group was missing the pulse value.

$\dagger$ Two patients in the nonneurosurgical group and 1 in the neurosurgical group were missing BP data.

$\ddagger$ One patient in the nonneurosurgical group and 2 in the neurosurgical group were missing respiratory data.

$\S$ Eight patients in the nonneurosurgical group and 7 in the neurosurgical group were missing temperature data. 
TABLE 2. Subdural hematoma characteristics by neurosurgical intervention group

\begin{tabular}{|c|c|c|c|c|}
\hline Hemorrhage Characteristic & Overall, $N=176$ & No Neurosurgical Intervention, $n=148$ & Neurosurgical Intervention, $n=28$ & $p$ Value \\
\hline \multicolumn{5}{|l|}{ Location } \\
\hline Frontal lobe & $119(68 \%)$ & $91(61 \%)$ & $28(100 \%)$ & $<0.001$ \\
\hline Temporal lobe & $84(48 \%)$ & $63(43 \%)$ & $21(75 \%)$ & 0.002 \\
\hline Parietal lobe & $86(49 \%)$ & $59(40 \%)$ & $27(96 \%)$ & $<0.001$ \\
\hline Occipital lobe & $37(21 \%)$ & $24(16 \%)$ & $13(46 \%)$ & $<0.001$ \\
\hline Tentorium & $38(22 \%)$ & $35(24 \%)$ & $3(11 \%)$ & 0.13 \\
\hline Falx & $61(35 \%)$ & $57(39 \%)$ & $4(14 \%)$ & 0.01 \\
\hline Total lobes involved in bleed & & & & $<0.001$ \\
\hline 0 & $39(22 \%)$ & $39(26 \%)$ & 0 & \\
\hline 1 & $39(22 \%)$ & $38(26 \%)$ & $1(4 \%)$ & \\
\hline 2 & $37(21 \%)$ & $31(21 \%)$ & $6(21 \%)$ & \\
\hline 3 & $31(18 \%)$ & $23(16 \%)$ & $8(29 \%)$ & \\
\hline 4 & $30(17 \%)$ & $17(11 \%)$ & $13(46 \%)$ & \\
\hline AOC bleed & $36(20 \%)$ & $15(10 \%)$ & $21(75 \%)$ & $<0.001$ \\
\hline IVH present & $2(1 \%)$ & $1(1 \%)$ & $1(4 \%)$ & 0.29 \\
\hline Mass effect present & $60(34 \%)$ & $38(26 \%)$ & $22(79 \%)$ & $<0.001$ \\
\hline Midline shift & & & & $<0.001$ \\
\hline None or no mention & $129(73 \%)$ & $126(85 \%)$ & $3(11 \%)$ & \\
\hline$\leq 5 \mathrm{~mm}$ & $23(13 \%)$ & $18(12 \%)$ & $5(18 \%)$ & \\
\hline$>5 \mathrm{~mm}$ & $24(14 \%)$ & $4(3 \%)$ & $20(71 \%)$ & \\
\hline
\end{tabular}

Boldface type indicates statistical significance.

None of the 19 patients with falx-only SDHs, and none of the 9 patients with tentorial-only SDHs required a neurosurgical procedure. Furthermore, the neurosurgical intervention group had significantly more patients with hemorrhages involving multiple lobes; there was a significant, positive trend between number of lobes involved and the proportion of patients in the neurosurgical intervention group (Cochran-Armitage trend test, p-trend < 0.001). There was also a positive correlation between the number of lobes involved and the maximum SDH length (Pearson correlation 0.70, $\mathrm{p}<0.001)$. The presence of an AOC SDH, mass effect, and a midline shift $>5 \mathrm{~mm}$ was significantly associated with the neurosurgical intervention group.
There was no significant difference between study groups in the proportion with IVH.

There were significant differences between neurosurgical intervention groups vis-à-vis maximum hemorrhage length and thickness (Table 3). Nine patients were not included in the measurement analyses because they suffered isolated tentorial SDHs. The neurosurgical intervention group had an average maximum SDH length that was 60.9 $\mathrm{mm}$ longer than that of the nonneurosurgical intervention group. Additionally, the neurosurgical intervention group had an average maximum SDH thickness that was 13.4 $\mathrm{mm}$ thicker than that of the nonneurosurgical intervention group. The smallest SDH in the intervention group,

TABLE 3. Subdural hematoma measurements by neurosurgical intervention group

\begin{tabular}{cllllr}
\hline $\begin{array}{c}\text { Hemorrhage } \\
\text { Characteristic }\end{array}$ & Overall, $\mathrm{N}=167^{*}$ & $\begin{array}{c}\text { No Neurosurgical } \\
\text { Intervention, } \mathrm{n}=148\end{array}$ & $\begin{array}{c}\text { Neurosurgical } \\
\text { Intervention, } \mathrm{n}=28\end{array}$ & $\begin{array}{c}\text { Mean Difference, } \\
95 \% \mathrm{Cl}\end{array}$ & $\begin{array}{c}\mathrm{p} \\
\text { Value }\end{array}$ \\
\hline Max length, $\mathrm{mm}$ & & & & & \\
\hline Mean (SD) & $99.6(55.7)$ & $89.4(51.84)$ & $150.3(46.6)$ & $60.9,40.0-81.8$ & $<0.001$ \\
\hline Median (IQR) & $94.5(51.3,147.0)$ & $79.5(45.9,126.2)$ & $158.0(125.9,187.4)$ & & $<0.001$ \\
\hline Min, max & $11.7,221.0$ & $11.7,221.0$ & $23.1,207.5$ & & \\
\hline Max thickness, mm & & & & & \\
\hline Mean (SD) & $9.5(7.1)$ & $7.3(4.2)$ & $20.7(7.9)$ & $13.4,10.2-16.5$ & $<0.001$ \\
\hline Median (IQR) & $7.0(4.8,12.2)$ & $6.1(4.5,9.2)$ & $19.9(14.0,27.1)$ & & $<0.001$ \\
\hline Min, max & $1.7,35.3$ & $1.7,28.5$ & $6.3,35.3$ & & \\
\hline
\end{tabular}

Max = maximum; $\min =$ minimum .

Boldface type indicates statistical significance.

* Nine patients suffered a tentorial-only SDH and did not contribute any measurements to the overall study population. 


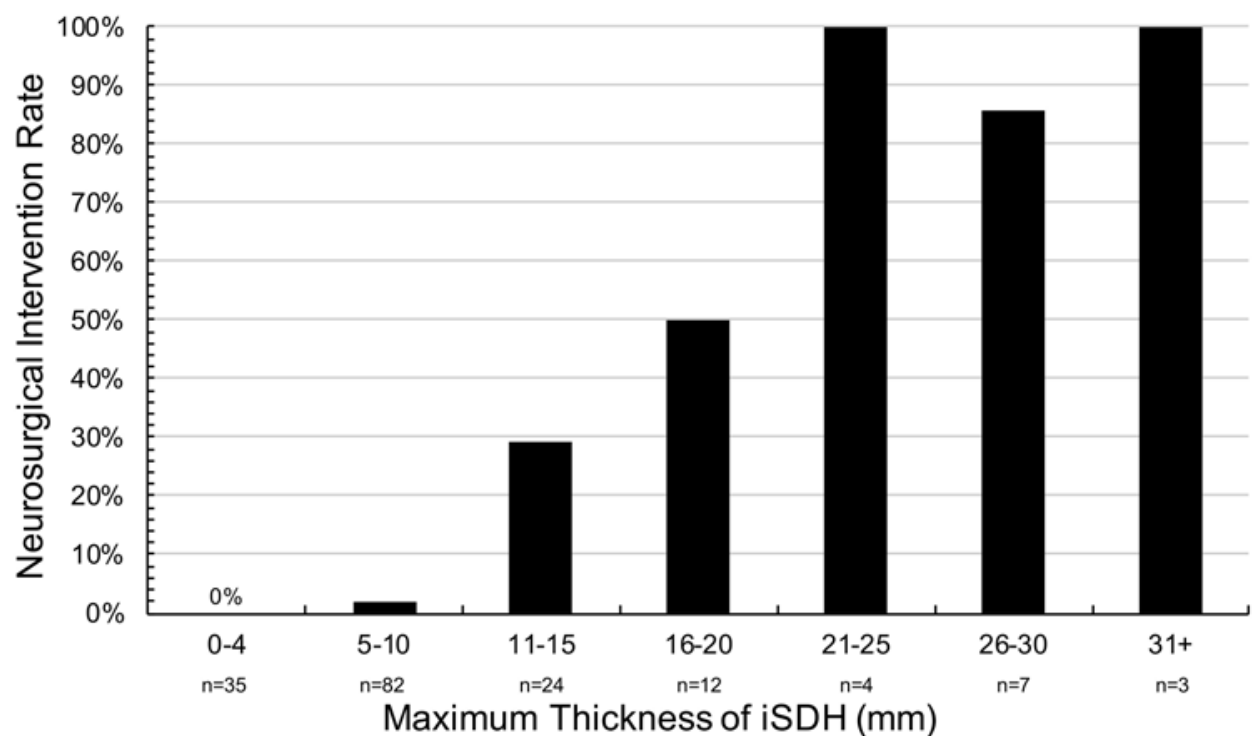

FIG. 1. Bar graph showing actual rate of neurosurgical intervention by maximum thickness of SDH. This figure shows the increasing rate of neurosurgical intervention associated with increasing maximum thickness of SDHs.

on initial presenting $\mathrm{CT}$, was $6.3 \mathrm{~mm}$ in thickness. Figure 1 shows the actual rate of neurosurgical intervention based on maximum SDH thickness categories. There was a significant trend between increasing SDH thickness and neurosurgical rate $(\mathrm{p}$-trend $<0.001)$.

We assessed the association between independent variables and neurosurgical intervention based on a multivariable logistic regression model. All variables with a p value $\leq 0.20$ in Tables 1 and 2 were included in the model, except for mass effect, midline shift, and number of lobes

TABLE 4. Logistic regression models examining odds of neurosurgical intervention

\begin{tabular}{|c|c|c|}
\hline Variable & $\begin{array}{l}\text { Unadjusted OR } \\
\qquad(95 \% \mathrm{Cl})\end{array}$ & $\begin{array}{l}\text { Adjusted OR } \\
(95 \% \mathrm{Cl})^{*}\end{array}$ \\
\hline \multicolumn{3}{|l|}{ Head AIS score $†$} \\
\hline $2 \& 3$ & $0.30(0.09-1.04)$ & \\
\hline 4 & $0.11(0.02-0.50)$ & \\
\hline 5 & Reference & \\
\hline Postinjury LOC & $0.15(0.04-0.53)$ & \\
\hline AOC bleed & 26.6 (9.71-72.91) & $3.07(0.80-11.85)$ \\
\hline Mass effect present & $10.61(4.00-28.14)$ & \\
\hline \multicolumn{3}{|l|}{ Midline shift } \\
\hline None or no mention & Reference & \\
\hline$\leq 5 \mathrm{~mm}$ & $11.67(2.57-53.03)$ & \\
\hline$>5 \mathrm{~mm}$ & $210.00(43.71$ to $>99.99)$ & \\
\hline $\begin{array}{l}\text { Max length: } 10-m m \\
\text { increase }\end{array}$ & $1.25(1.14-1.37)$ & \\
\hline $\begin{array}{l}\text { Max thickness: 1-mm } \\
\text { increase }\end{array}$ & $1.39(1.23-1.56)$ & $1.32(1.16-1.50)$ \\
\hline
\end{tabular}

${ }^{*}$ AUROC curve $=0.94 ;$ Hosmer-Lemeshow goodness-of-fit $p$ value $=0.98$. $\dagger$ Scores of 2 and 3 were collapsed to avoid quasi-complete separation of data points in logistic regression models. involved because of their collinearity with hemorrhage length and thickness; head AIS score was not made available to the model because this information is not calculated until after patient discharge, and for patients with an $\mathrm{SDH}$ it is essentially determined by the SDH thickness. Our final model only included two variables: maximum hemorrhage thickness and the presence of an AOC SDH (Table 4). In the final model, hemorrhage thickness was the most important variable; there was no significant interaction between hemorrhage thickness and the presence of an AOC hemorrhage (interaction $\mathrm{p}=0.25$ ). The model resulted in good discrimination and calibration, per an AUROC curve of 0.94 (95\% CI 0.90-0.97), and a HosmerLemeshow goodness-of-fit $\mathrm{p}$ value of 0.98 . Per our data, after adjusting for the presence of an AOC hemorrhage, for every 1-mm increase in the thickness of an iSDH in a patient with mTBI, the odds of a neurosurgical intervention increase by $32 \%$.

Nine patients did not have follow-up imaging. Of the 167 patients with follow-up imaging, 90\% had SDHs that were stable, resolving, or had completely resolved; $10 \%$ had SDHs that increased in size. There were no significant differences in the mortality rates or hospital dispositions between the neurosurgical intervention groups (Table 5). However, the group that received neurosurgical intervention had a significantly longer median hospital LOS compared to the nonneurosurgical intervention group. Furthermore, neither maximum hemorrhage thickness nor length was significantly associated with increased odds of in-hospital mortality ( $\mathrm{OR}_{\text {thickness }} 1.05$ [95\% CI 0.96-1.14]; $\mathrm{OR}_{\text {length }}$ 1.10 [95\% CI 0.96-1.26]). The only radiographic characteristic that was significantly associated with in-hospital mortality was the presence of mass effect (OR 12.78 [95\% CI 1.50-108.73]).

\section{Discussion}

The objective of this study was to assess the relation- 
TABLE 5. Outcomes in patients with $\mathrm{mTBI}$ and iSDH

\begin{tabular}{|c|c|c|c|c|}
\hline Outcomes & Overall, $\mathrm{N}=176$ & No Neurosurgical Intervention, $n=148$ & Neurosurgical Intervention, $n=28$ & $\mathrm{p}$ Value \\
\hline In-hospital mortality & $7(4 \%)$ & $4(3 \%)$ & $3(11 \%)$ & 0.08 \\
\hline \multicolumn{5}{|l|}{ Hospital LOS, days } \\
\hline Median (IQR) & $3(2,5)$ & $3(2,5)$ & $5(3,9)$ & 0.002 \\
\hline Min, $\max$ & 0,40 & 0,17 & 1,40 & \\
\hline Hospital disposition & & & & 0.11 \\
\hline Home/home health & $101(57 \%)$ & $90(61 \%)$ & $11(40 \%)$ & \\
\hline Rehab/skilled nursing facility & $54(31 \%)$ & $42(28 \%)$ & $12(43 \%)$ & \\
\hline Acute care facility & $1(1 \%)$ & $1(1 \%)$ & 0 & \\
\hline Discharged against medical advice & $3(2 \%)$ & $3(2 \%)$ & 0 & \\
\hline Hospice & $10(6 \%)$ & $8(5 \%)$ & $2(7 \%)$ & \\
\hline Dead & $7(4 \%)$ & $4(3 \%)$ & $3(11 \%)$ & \\
\hline
\end{tabular}

Boldface type indicates statistical significance. Unless indicated otherwise, the values are expressed as the number of patients (\%).

ship between admission quantitative hemorrhage characteristics of iSDHs in patients with mTBI and the risk for subsequent neurosurgical intervention. Our study successfully identified many radiological and clinical characteristics associated with neurosurgical intervention. Regarding hemorrhage characteristics, the location of the hemorrhage, number of lobes involved, presence of an AOC hemorrhage, mass effect, and midline shift were all significantly associated with neurosurgical intervention. More importantly, maximum hemorrhage thickness and length were also significantly associated with neurosurgical intervention in univariate analyses.

This is the first study to our knowledge to examine the association between quantitative measurements of iSDH and subsequent neurosurgical intervention in patients with mTBI. We created a model specifically for this injury, which quantified the odds of a neurosurgical intervention by using two variables that are readily accessible on admission radiological imaging. The two most important variables associated with the need for a neurosurgical intervention in our study population were maximum thickness of the iSDH, and whether or not the SDH was AOC (although of these the maximum thickness was the more important variable).

Our model had an AUROC curve (0.94) larger than many published predictive models in the TBI population. ${ }^{6,7,21-23,27,32,33,35} \mathrm{We}$ believe the high discrimination power of our model was attributable to the granularity and volume of data collected from head CT images, and the narrow focus on this subpopulation of patients with mTBI and iSDH. By collecting hematoma thickness and length to an accuracy of one-tenth of a millimeter, in addition to collecting a panoply of hemorrhage characteristics, including AOC and midline shift, we could assess the importance of each characteristic, and retain the variables that best explained the risk for neurosurgical intervention.

The benefits stemming from our incorporation of detailed radiological data into our predictive models are mirrored in other recent studies in this field. ${ }^{13,27}$ A recent study by Shih and colleagues examined neurosurgical interventions in 347 patients with mTBI and $\mathrm{ICH}$, and incorporated volumetric hemorrhage information. ${ }^{27}$ In their study,
Shih et al. calculated the total volume for each EDH, SDH, and intraparenchymal contusion, and found that only the volume of EDH was significantly different between neurosurgical intervention groups in univariate analysis. The study was limited to the inclusion of only one independent predictor in the final logistic regression model due to limited outcome observations $(n=13)$. The volume of the EDH was identified as the only independent variable predicting subsequent neurosurgical intervention (AUROC curve $0.92,95 \%$ CI $0.80-1.00$ ). Although it achieved a high AUROC curve value, the study only included 6 patients with an EDH, and had an unusually low neurosurgical intervention rate for SDHs (3.6\%), limiting the generalizability of the results and the neurosurgical practices. Another study in an mTBI population corroborated SDH and EDH sizes as being independent predictors of neurosurgical intervention..$^{13}$ However, there is ambiguity surrounding the definition for the term "hemorrhage size" in the study, and there is no reporting of the discrimination or goodness-of-fit measures for their final predictive model, making model assessment difficult.

A main limitation of the TBI literature is the lack of studies examining neurosurgical interventions in the mTBI population. ${ }^{15,24}$ Understanding the relationship between neurosurgical intervention and characteristics of ICH in the mTBI population can improve upon current decision criteria. Multiple decision algorithms predicting neurosurgical interventions have defined hemorrhage sizes as "mild" or "clinically unimportant"-not through an analysis of risk and hemorrhage characteristics, but from anecdote and surveys. The Brain Injury Guidelines study categorizes SDHs as "minor head injury" if they are $\leq 4$ mm thick. ${ }^{12}$ Huynh et al. defined SDHs $<3 \mathrm{~mm}$ thick as "clinically unimportant" and based the definition on the presentation and outcomes of no more than 10 patients with SDHs, for which there was no detailed reporting of hemorrhage characteristics. ${ }^{8}$ Additionally, the Canadian CT Head Rule defines SDHs as "clinically unimportant" if the patient is neurologically intact and has a hemorrhage < $4 \mathrm{~mm}$ thick..$^{30}$ This determination was not made based on an analysis of quantitative hemorrhage data; instead, it was based on a survey of 129 academic physicians. Although 
experienced practicing neurosurgeons have believed this opinion to be accurate for many years, our quantitative analysis has finally given a firm foundation to this assumption. In general, we should shy away from categorizing hemorrhages as "minor" or "unimportant," because these definitions probably underestimate the variance in risk of poor patient outcomes.

Most importantly, and not surprisingly, we found that no patients with SDHs $<5 \mathrm{~mm}$ in maximum thickness, or with falx-only or tentorium-only SDHs required neurosurgical intervention. Our data suggest that AOC SDH in patients with mTBI might carry a higher risk of requiring neurosurgical intervention, independent of the size of the hematoma. This is probably secondary to AOC SDH presenting as mTBI even when the SDH is significantly larger, because most of the SDH volume is usually chronic and accumulates or increases slowly over time, allowing the patient and the brain to accommodate the larger volume with later and fewer clinical effects. Therefore, the presence of an AOC SDH-as well as chronic and subacute SDHs - must be incorporated into a clinical decision, and in the development or use of predictive models. Regardless, the clinical importance of a hemorrhage should, at a minimum, incorporate a quantitative assessment of risk for neurosurgical intervention, and should also include quantitative radiological and clinical information.

Although a change in GCS score is used to identify neurological deterioration, which is often an indication for a neurosurgical intervention, admission GCS score has been repeatedly shown to be a poor determinant of subsequent neurosurgical intervention; our study lends support to this conclusion. 3,27,36 This reoccurring observation should not be unexpected because the GCS was designed to reflect differences in the risk for mortality, not neurosurgical intervention. When we examined the interaction of GCS score and hemorrhage thickness in a multivariable logistic regression model, the odds of neurosurgical intervention for each millimeter increase in hemorrhage thickness was similar for each admission GCS group (p-interaction 0.19). Therefore, the data suggest the probability of neurosurgical intervention was driven by hemorrhage thickness, rather than GCS score. However, 74\% of our study population presented with a GCS score of 15 ; thus, it is inappropriate to definitively claim a lack of an interaction between risk of hemorrhage thickness and admission GCS score.

Although in-hospital mortality was a secondary outcome variable, our study was not powered to appropriately investigate the associations between hemorrhage characteristics and mortality. With only 7 in-hospital deaths, we were precluded from adjusting for confounding factors such as age, comorbid factors, radiological factors, and other factors, including do-not-resuscitate orders and comfort care placement. As such, we would suggest extending caution when interpreting the mortality data. Nevertheless, our study supports the notion of a small rate of in-hospital mortality in this patient population with iSDHs and mild TBI, and no significant difference in the mortality rates between those who underwent and those who did not undergo neurosurgical interventions.

An interesting finding of our study is the ironic relationship between LOC and neurosurgical intervention. Our data show that patients who required neurosurgical intervention had a significantly lower rate of postinjury LOC compared to patients who did not require a neurosurgical intervention. A potential explanation for this paradoxical finding is a significant circular association between LOC, age, hemorrhage thickness, and neurosurgical intervention. Upon further investigation of our data, we discovered there was a significantly smaller proportion of older patients $(\geq$ 65 years) who suffered a postinjury LOC, compared to younger patients (18-64 years, $27 \%$ vs $59 \%$; p < 0.001$)$. Additionally, older patients had significantly thicker SDHs than did younger patients $\left(\mu_{\text {diff }}=2.84\right.$ [0.96]). To complete the circle of associations, our study concluded that thicker SDHs were more associated with neurosurgical interventions than thinner SDHs (Table 3). It has been documented that older age is associated with age-related cerebral atrophy, $25,26,31$ which is followed by an increased volume of intracranial CSF. ${ }^{16}$ It stands to reason that if older patients have a lower risk of suffering LOC after a mild TBI, then it is possible that their increased CSF volume might help decrease the internal forces exerted on the brain during a mild TBI, thus mitigating their risk of LOC. If this association holds true in further studies, then it seems age-related cerebral atrophy is the most likely driving force behind the associations between age and all three of our findings in older patients: a decreased occurrence of LOC, an increased risk of thicker SDHs, and a greater probability of requiring neurosurgical intervention in the setting of mild TBI.

Another possible explanation is that older patients might require a less impactful insult to their head to cause an ICH, compared to younger patients. Cerebral atrophy is known to lead to increased tension on the intracranial blood vessels extending through the meninges. Thus, if older patients are experiencing less force to the head than younger patients when sustaining an mTBI, they might also have a smaller chance of suffering an LOC. Although this odd relationship could be a result of chance, we encourage other researchers to investigate this phenomenon and attempt to duplicate our results. It would be important to replicate these findings in patients with concussion-only head injuries, to mitigate the potential bias caused by force of injury and age-related risk of ICH.

The main limitation of our study is that it lacked sufficient patients to both train and test the validity of our final logistic regression model. Thus, our model must be validated in a larger, separate population prior to our recommending its use. Despite this limitation, we believe it is important to present our preliminary model showing the benefits of incorporating quantitative characteristics of an ICH into a predictive model for neurosurgical intervention. Although some predictive models have incorporated the ICH volume, we instead chose to describe each SDH in only two dimensions-length and thickness. Our reasoning behind obtaining maximum length and thickness instead of hemorrhage volume was due to time concerns and the limited availability of advanced software. Calculating the volume of a hematoma is more time-consuming, and generally requires coronal images, or counting of slices and multiplying by slice thickness, compared to the rapid simplicity of calculating the maximum thickness. Addi- 
tionally, volume calculations are not available on all imaging software. If available, volume calculations require the user to manually paint the hemorrhage area on each image slice individually, and are especially difficult for crescentshaped SDHs extending over a large portion of the cerebral convexity. Most referring facilities without neurosurgical capabilities (i.e., those that would benefit most from the use of a simple scoring tool for SDHs) may not have the time, technology, or training to accurately calculate hematoma volumes. On the other hand, the time necessary for measuring the maximum hematoma thickness is an order of magnitude lower, taking seconds, not minutes.

Another limitation of this study was that we excluded patients with skull fractures. This exclusion was necessary because the initial means of identifying potentially eligible patients for this study was via ICD-9 codes. Because ICD9 codes for a skull fracture and an ICH do not distinguish between $\mathrm{EDH}, \mathrm{SDH}$, or $\mathrm{SAH}$, we were unable to identify the SDH-only patients in this cohort. This exclusion would be unnecessary if using ICD-10 codes, due to its segregation of skull fracture codes from ICH codes. Future studies should seek to replicate our findings in the mTBI population with iSDH, as well as in other ICH types and in populations with non-mTBI.

\section{Conclusions}

We have demonstrated the utility of incorporating the radiographic characteristics of an iSDH into a model assessing the odds of neurosurgical intervention for patients with mTBI and without coagulopathy. Our initial model was highly accurate, and used variables and measurements easily obtained via standard imaging techniques. We identified that the presence of an AOC SDH and the maximum hematoma thickness were the two most important variables in predicting the odds of neurosurgical intervention in this population. We believe these data, once validated, will help inform the development of new management guidelines for patients with mild TBI and SDH.

\section{References}

1. Borczuk P: Predictors of intracranial injury in patients with mild head trauma. Ann Emerg Med 25:731-736, 1995

2. Carlson AP, Ramirez P, Kennedy G, McLean AR, MurrayKrezan C, Stippler M: Low rate of delayed deterioration requiring surgical treatment in patients transferred to a tertiary care center for mild traumatic brain injury. Neurosurg Focus 29(5):E3, 2010

3. Ditty BJ, Omar NB, Foreman PM, Patel DM, Pritchard PR, Okor MO: The nonsurgical nature of patients with subarachnoid or intraparenchymal hemorrhage associated with mild traumatic brain injury. J Neurosurg 123:649-653, 2015

4. Esposito TJ, Reed RL II, Gamelli RL, Luchette FA: Neurosurgical coverage: essential, desired, or irrelevant for good patient care and trauma center status. Ann Surg 242:364374, 2005

5. Faul MD, Xu L, Wald MM, Coronado VG: Traumatic Brain Injury in the United States. Emergency Department Visits, Hospitalizations, and Deaths, 2002-2006. Atlanta: Centers for Disease Control and Prevention, National Center for Injury Prevention and Control, 2010

6. Hukkelhoven CW, Rampen AJ, Maas AI, Farace E, Habbema JD, Marmarou A, et al: Some prognostic models for traumat- ic brain injury were not valid. J Clin Epidemiol 59:132-143, 2006

7. Hukkelhoven CW, Steyerberg EW, Habbema JD, Farace E, Marmarou A, Murray GD, et al: Predicting outcome after traumatic brain injury: development and validation of a prognostic score based on admission characteristics. J Neurotrauma 22:1025-1039, 2005

8. Huynh T, Jacobs DG, Dix S, Sing RF, Miles WS, Thomason $\mathrm{MH}$ : Utility of neurosurgical consultation for mild traumatic brain injury. Am Surg 72:1162-1165, n1166-n1167, 2006

9. Jagoda AS, Bazarian JJ, Bruns JJ Jr, Cantrill SV, Gean AD, Howard PK, et al: Clinical policy: neuroimaging and decisionmaking in adult mild traumatic brain injury in the acute setting. Ann Emerg Med 52:714-748, 2008

10. Jeret JS, Mandell M, Anziska B, Lipitz M, Vilceus AP, Ware JA, et al: Clinical predictors of abnormality disclosed by computed tomography after mild head trauma. Neurosurgery 32:9-16, 1993

11. Joseph B, Aziz H, Sadoun M, Kulvatunyou N, Tang A, O'Keeffe T, et al: The acute care surgery model: managing traumatic brain injury without an inpatient neurosurgical consultation. J Trauma Acute Care Surg 75:102-105, 2013

12. Joseph B, Friese RS, Sadoun M, Aziz H, Kulvatunyou N, Pandit V, et al: The BIG (brain injury guidelines) project: defining the management of traumatic brain injury by acute care surgeons. J Trauma Acute Care Surg 76:965-969, 2014

13. Joseph B, Pandit V, Aziz H, Kulvatunyou N, Zangbar B, Green DJ, et al: Mild traumatic brain injury defined by Glasgow Coma Scale: Is it really mild? Brain Inj 29:11-16, 2015

14. Levy AS, Orlando A, Salottolo K, Mains CW, Bar-Or D: Outcomes of a nontransfer protocol for mild traumatic brain injury with abnormal head computed tomography in a rural hospital setting. World Neurosurg 82:e319-e323, 2014

15. Maas AI, Lingsma HF, Roozenbeek B: Predicting outcome after traumatic brain injury. Handb Clin Neurol 128:455474, 2015

16. MacKenzie JD, Siddiqi F, Babb JS, Bagley LJ, Mannon LJ, Sinson GP, et al: Brain atrophy in mild or moderate traumatic brain injury: a longitudinal quantitative analysis. AJNR Am J Neuroradiol 23:1509-1515, 2002

17. Miller EC, Holmes JF, Derlet RW: Utilizing clinical factors to reduce head CT scan ordering for minor head trauma patients. J Emerg Med 15:453-457, 1997

18. National Center for Injury Prevention and Control: Report to Congress on Mild Traumatic Brain Injury in the United States: Steps to Prevent a Serious Public Health Problem. Atlanta: Centers for Disease Control and Prevention, 2003 (https://www.cdc.gov/traumaticbraininjury/pdf/mtbireport-a. pdf) [Accessed February 15, 2018]

19. Nishijima DK, Sena MJ, Holmes JF: Identification of lowrisk patients with traumatic brain injury and intracranial hemorrhage who do not need intensive care unit admission. J Trauma 70:E101-E107, 2011

20. Orlando A, Levy AS, Carrick MM, Tanner A, Mains CW, Bar-Or D: Epidemiology of mild traumatic brain injury with intracranial hemorrhage: focusing predictive models for neurosurgical intervention. World Neurosurg 107:94-102, 2017

21. Papa L, Lewis LM, Falk JL, Zhang Z, Silvestri S, Giordano $\mathrm{P}$, et al: Elevated levels of serum glial fibrillary acidic protein breakdown products in mild and moderate traumatic brain injury are associated with intracranial lesions and neurosurgical intervention. Ann Emerg Med 59:471-483, 2012

22. Papa L, Lewis LM, Silvestri S, Falk JL, Giordano P, Brophy GM, et al: Serum levels of ubiquitin C-terminal hydrolase distinguish mild traumatic brain injury from trauma controls and are elevated in mild and moderate traumatic brain injury patients with intracranial lesions and neurosurgical intervention. J Trauma Acute Care Surg 72:1335-1344, 2012 
23. Perel P, Arango M, Clayton T, Edwards P, Komolafe E, Poccock $S$, et al: Predicting outcome after traumatic brain injury: practical prognostic models based on large cohort of international patients. BMJ 336:425-429, 2008

24. Perel P, Edwards P, Wentz R, Roberts I: Systematic review of prognostic models in traumatic brain injury. BMC Med Inform Decis Mak 6:38, 2006

25. Peters R: Ageing and the brain. Postgrad Med J 82:84-88, 2006

26. Scahill RI, Frost C, Jenkins R, Whitwell JL, Rossor MN, Fox NC: A longitudinal study of brain volume changes in normal aging using serial registered magnetic resonance imaging. Arch Neurol 60:989-994, 2003

27. Shih FY, Chang HH, Wang HC, Lee TH, Lin YJ, Lin WC, et al: Risk factors for delayed neuro-surgical intervention in patients with acute mild traumatic brain injury and intracranial hemorrhage. World J Emerg Surg 11:13, 2016

28. Sifri ZC, Homnick AT, Vaynman A, Lavery R, Liao W, Mohr A, et al: A prospective evaluation of the value of repeat cranial computed tomography in patients with minimal head injury and an intracranial bleed. J Trauma 61:862-867, 2006

29. Stiell IG, Clement CM, Rowe BH, Schull MJ, Brison R, Cass D, et al: Comparison of the Canadian CT Head Rule and the New Orleans Criteria in patients with minor head injury. JAMA 294:1511-1518, 2005

30. Stiell IG, Wells GA, Vandemheen K, Clement C, Lesiuk H, Laupacis A, et al: The Canadian CT Head Rule for patients with minor head injury. Lancet 357:1391-1396, 2001

31. Svennerholm L, Boström K, Jungbjer B: Changes in weight and compositions of major membrane components of human brain during the span of adult human life of Swedes. Acta Neuropathol 94:345-352, 1997

32. Sweeney TE, Salles A, Harris OA, Spain DA, Staudenmayer KL: Prediction of neurosurgical intervention after mild traumatic brain injury using the National Trauma Data Bank. World J Emerg Surg 10:23, 2015

33. Velmahos GC, Gervasini A, Petrovick L, Dorer DJ, Doran ME, Spaniolas K, et al: Routine repeat head CT for minimal head injury is unnecessary. J Trauma 60:494-501, 2006

34. Washington CW, Grubb RL Jr: Are routine repeat imaging and intensive care unit admission necessary in mild traumatic brain injury? J Neurosurg 116:549-557, 2012

35. Weinberg AM, Castellani C: Role of neuroprotein S-100B in the diagnostic of pediatric mild brain injury. Eur J Trauma Emerg Surg 36:318-324, 2010

36. Wu C, Orringer DA, Lau D, Fletcher JJ: Cumulative incidence and predictors of neurosurgical interventions following nonsevere traumatic brain injury with mildly abnormal head imaging findings. J Trauma Acute Care Surg 73:12471253,2012

\section{Disclosures}

The authors report no conflict of interest concerning the materials or methods used in this study or the findings specified in this paper.

\section{Author Contributions}

Conception and design: Orlando, Levy. Acquisition of data: Orlando, Levy. Analysis and interpretation of data: Orlando, Levy, Rubin, Tanner, Lieser. Drafting the article: Bar-Or, Orlando, Levy, Tanner, Carrick, Hamilton, Mains. Critically revising the article: all authors. Reviewed submitted version of manuscript: all authors. Statistical analysis: Orlando. Administrative/technical/ material support: Bar-Or, Orlando, Hamilton, Mains. Study supervision: Bar-Or.

\section{Supplemental Information}

\section{Companion Papers}

Orlando A, Levy AS, Rubin BA, Tanner A, Carrick MM, Lieser $\mathrm{M}$, et al: Isolated subdural hematomas in mild traumatic brain injury. Part 2: a preliminary clinical decision support tool for neurosurgical intervention. DOI: 10.3171/2018.1.JNS171906.

\section{Previous Presentations}

Portions of this work were presented at the 2017 American Association for the Surgery of Trauma meeting in Baltimore, Maryland, on September 13, 2017.

\section{Correspondence}

David Bar-Or: Swedish Medical Center, Englewood, CO. dbaror@ ampiopharma.com. 